Original Paper http://ajol.info/index.php/ijbcs http://indexmedicus.afro.who.int

\title{
Analyse socioéconomique du système de commercialisation de la banane plantain dans la région de l'Ouest Cameroun
}

\author{
Guillaume Hensel FONGANG FOUEPE ${ }^{1 *}$, Achile BIKOI², \\ Denis Pompidou FOLEFACK ${ }^{2}$, Idriss TIECHE ${ }^{1}$ et Pascal NOUPADJA ${ }^{2}$ \\ ${ }^{1}$ Faculté d'Agronomie et des Sciences Agricoles (FASA), Département de Socio-Economie Rurale et \\ Vulgarisation Agricole, Université de Dschang, B.P 222 Dschang, Cameroun. \\ ${ }^{2}$ Centre Africain de Recherches sur Bananiers et Plantains (CARBAP), B.P. 832 Douala, Cameroun. \\ *Auteur correspondant ; E-mail : guillaumefongang@yahoo.fr, Téléphone : (+ 237) 696206204
}

\section{RESUME}

La commercialisation de la banane-plantain occupe une place prépondérante dans les flux des produits agricoles. Cette étude met en exergue le fonctionnement des différents systèmes de commercialisation du produit et leurs rentabilités économiques dans la région de l'Ouest Cameroun. Des enquêtes ont été menées auprès de 167 acteurs de la filière (33 producteurs, 50 collecteurs, 25 grossistes, 44 détaillants et 15 transporteurs) dans la région. Les résultats montrent que les catégories d'acteurs impliquées dans le système de commercialisation de la banane plantain ont des profils variés. Les producteurs sont majoritairement des hommes (75\%) à 55\% âgés entre 50 et 70 ans, les transporteurs n'ont pas plus de 49 ans, les commerçants (collecteurs, grossistes, détaillants) sont essentiellement des femmes (respectivement $84 \%, 88 \%$ et $75 \%$ ). L'étude a permis de distinguer deux types de marchés : les marchés ruraux d'approvisionnement et les marchés urbains d'écoulement. Deux grands axes de commercialisation de la banane-plantain ont été identifiés, l'axe Pont du Noun-Kouoptamo-Bafoussam et l'axe Galim-Mbouda-Bamenda. Le plantain passe par plusieurs intermédiaires qui varient en fonction du type de circuit (circuit de vente directe, circuit court, circuit long). L'activité de commercialisation est une activité très rentable et le niveau de rentabilité est fonction du type d'acteurs et des contraintes rencontrées.

(c) 2019 International Formulae Group. All rights reserved

Mots clés : Banane-plantain, commercialisation, circuits, intermédiaires, marchés, Ouest-Cameroun.

\section{Socio economic analysis of the banana-plantain marketing system in the Western region of Cameroon}

\begin{abstract}
The commercialization of banana-plantain plays a predominant role in the flow of agricultural products. The study highlights the functioning of the various banana-plantain marketing systems and their economic viability in the West region of Cameroon. Surveys were carried out among the actors in the sector (33 producers, 50 collectors, 25 wholesalers, 44 retailers and 15 transporters) in the region. The results show that the categories of actors involved in the banana plantain marketing system are varied. Producers are mostly male $(75 \%)$ with $55 \%$, ranging between 50 and 70 years, transporters do not exceed 49 years, traders
\end{abstract}


(collectors, wholesalers, retailers) are mainly women $84 \%, 88 \%$ and $75 \%$ respectively. The study distinguishes between two types of markets: rural procurement markets (the production market and the collection market) and urban markets. There are two major marketing axes for banana-plantain: the "Pont du Noun-KouoptamoBafoussam" axis and the "Galim-Mbouda-Bamenda" axis. Before reaching the consumer, plantain passes through several intermediaries who vary according to the type of circuit (direct sales circuit, short distance circuit, long distance circuit). The constraints are physical and human and vary according to the category of actors.

(C) 2019 International Formulae Group. All rights reserved

Keywords: Banana-plantain, commercialization, circuit, intermediaries, markets, west-Cameroon.

\section{INTRODUCTION}

La banane-plantain occupe une place importante dans l'alimentation de la population camerounaise. Elle est une ressource indispensable pour les acteurs de cette filière. Du fait de son importance, la demande en banane-plantain est en pleine croissance au Cameroun en relation avec la pression démographique, l'accroissement de la population urbaine et grâce à ses diverses formes de consommation (Nkendah et Temple, 2003; Nkendah et Akyeampong, 2003 ; Nkendah et al., 2007 ; Wandji, 2015 ; Fongang et al., 2016 ; Folefack et al., 2017).

L'importance de ce produit est tant perceptible sur le plan national, que continental. Dans d'autres pays africains, certaines études sont allées un peu plus loin en mettant en lumière les produits et sousproduits de la filière, mais aussi leurs propriétés génétiques et l'amélioration des techniques de conservation (Lepengue et al 2010 ; Bouafou et al., 2012, Odah et al., 2013).

$\mathrm{Au}$ Cameroun, plusieurs facteurs limitent la consommation de cette denrée dans les grandes villes camerounaises, notamment : le prix élevé du plantain, l'instabilité de l'offre dans les marchés, la mauvaise organisation des marchés et des circuits de commercialisation, etc. En effet, les systèmes d'approvisionnement et de distribution des produits alimentaires dans les pays en développement sont inefficients compte tenu de leur caractère informel. De fait, l'approvisionnement des marchés urbains en vivres est parfois insuffisant (en quantité et en qualité) et très souvent sujet à de fortes variations saisonnières. Ces contraintes sont sensiblement les mêmes dans toute la partie méridionale du Cameroun où la bananeplantain est cultivée, mais leur intensité varie d'une zone agro écologique à l'autre (Fongang et al., 2016 ; Folefack et al., 2017).

En effet, la banane-plantain est un produit vital pour les pays en développement. Elle est un élément de base essentiel de l'alimentation dans des nombreux pays en développement, au même titre que le blé, le riz ou le maïs; d'où son importance en termes de sécurité alimentaire. Elle sert d'aliment de base à 400 millions de personnes dans le monde (FAOSTAT, 2011) et constitue un pilier important pour la sécurité alimentaire au Cameroun. Déjà en 2012, cette filière rassemblait plus de 700000 acteurs dont $92 \%$ étaient des petits producteurs. Elle contribue par ailleurs à $16 \%$ au revenu des producteurs en milieu paysan et de 4,5\% au PIB agricole (IRAD/CARBAP, 2012 ; MINADER, 2012).

La banane-plantain est présente dans presque toutes les exploitations familiales de la région de l'Ouest-Cameroun, du fait qu'elle occupe une place importante dans l'alimentation et l'économie des populations rurales de cette région. Sa production et sa commercialisation très intenses dans la région de l'Ouest (Zeugang, 2013) s'accompagnent de contraintes très variées. Les incertitudes sur les prix et le fonctionnement aléatoire de la commercialisation figurent parmi les plus importantes.

La présente étude a donc pour objectif de fournir un éclairage sur le fonctionnement des systèmes de commercialisation de la banane-plantain dans la région de l'Ouest 
Cameroun afin de ressortir les informations fiables susceptibles de permettre la formulation des recommandations pour une amélioration $\mathrm{du}$ fonctionnement de cette filière par les parties prenantes tels que les pouvoirs publics, les chercheurs, les organismes de développement et les producteurs.

\section{MATERIEL ET METHODES}

Cette étude a été menée dans le cadre du projet C2D/PAR Plantain de l'IRAD (Institut de recherche Agricole pour le Développement) et du CARBAP (Centre Africain de Recherche sur Bananiers et Plantains) dans deux grands bassins de production de la région de l'Ouest Cameroun que sont le Noun et le Bamboutos. Ces deux bassins ont été choisis du fait de l'importance de la production et de la commercialisation de la banane-plantain. Dans ces deux bassins, trois types de marché ont été pris en compte : deux marchés de production (Pont du Noun et Galim), deux marchés de collecte (Kouoptamo et Mbouda), et trois marchés de gros et détails (Bafoussam C, Foumbot et Bamenda).

Les données ont été collectées au moyen d'enquêtes par questionnaire et par guide d'entretien avec les personnes cibles entre juin et août 2015. Au total, 167 acteurs ont été enquêtés : 33 producteurs, 25 grossistes, 50 collecteurs, 44 détaillants et 15 transporteurs. Les données collectées portaient sur les caractéristiques sociodémographiques des acteurs, le rôle de chaque acteur, les axes et circuits de commercialisation, les stratégies des acteurs, les charges supportées par ces derniers, leurs marges bénéficiaires, les contraintes auxquelles ils font face, etc. La saisie et l'analyse des données collectées ont été réalisées au moyen des logiciels Excel et SPSS (Statistical Package for the Social Sciences) version 20.0. Les formules de calculs économiques ont été utilisées pour déterminer les marges des acteurs et comparer les performances des différents circuits de commercialisation identifiés.

\section{RESULTATS}

Caractéristiques sociodémographiques des acteurs impliqués

Les acteurs étudiés ici sont les producteurs, les collecteurs, les grossistes, les détaillants et les transporteurs. La production de la banane-plantain dans la région de l'Ouest Cameroun reste dominée par les hommes à $75,8 \%$, âgés pour la plupart d'au moins 50 ans $(54,5 \%)$. En ce qui concerne les collecteurs, la majorité est de sexe féminin soit $84 \%, 32 \%$ ont un âge compris entre 30 et 49 ans. Dans la vente en gros et détail, les femmes sont également plus présentes que les hommes. Les femmes grossistes et détaillantes impliquées dans la vente de plantain représentent respectivement $88 \%$ et $75 \%$ de l'ensemble des commerçantes présentes dans les marchés visités. Globalement, les commerçants concernés par cette étude sont dans une tranche d'âge comprise entre 40 et 49 ans. Le secteur du transport reste entièrement dominé par les hommes âgés entre 30 et 49 ans pour la majorité. Tous ces différents acteurs sont pour la plupart mariés avec comme niveau de scolarisation le primaire.

\section{Axes et circuits de commercialisation de la banane-plantain}

Le plantain est produit dans la région de l'Ouest Cameroun dans plusieurs bassins de production. Ces bassins sont repartis dans les 8 départements de la région. En raison de la périodicité (hebdomadaire), et du fait que 41 collecteurs sur 50 enquêtés et presque la totalité des grossistes s'y approvisionnent permanemment en banane plantain, deux (02) bassins ont été retenus (Bamboutos et Noun). En considérant pour ces deux bassins la circulation des produits en partant des principales zones de production jusqu'aux centres de consommation, il a été mis en évidence deux grands axes de commercialisation du plantain représentés sur la Carte 1. Ces axes sont les suivants :

- Axe 1 (A1):Galim $\rightarrow$ Mbouda $\rightarrow$ Bamenda

- Axe 2 (A2): Pont du Noun $\rightarrow$ Kouoptamo $\rightarrow$ Bafoussam 
La Figure 1 présente le schéma du circuit de commercialisation de la banane-plantain dans la région de l'Ouest Cameroun et met en évidence la nature des liens qui existe entre les différents acteurs impliqués.

\section{Les stratégies des acteurs impliqués Stratégies d'approvisionnement \\ Approvisionnement chez les collecteurs et grossistes}

Au niveau des collecteurs, les stratégies d'approvisionnement en banane-plantain varient selon les périodes et en fonction de la possession ou non d'un véhicule. Les prix des régimes de plantain diffèrent d'une période à une autre (période d'abondance et celle de pénurie). Pendant la période d'abondance, le producteur pratique un prix situé autour de 64 FCFA par kg de plantain, cependant, il peut atteindre une moyenne de 87 FCFA $/ \mathrm{kg}$ en période de pénurie. Les prix moyens d'achat pratiqués par les intermédiaires varient entre 60 et 157 FCFA $/ \mathrm{kg}$ respectivement en période d'abondance et de pénurie. Au final, le consommateur débourse en moyenne 265 FCFA pour le kilogramme de plantain. En période de pénurie, les collecteurs qui disposent d'un véhicule se rendent dans les plantations pour acheter les régimes bord champ si les routes sont praticables. Tandis que ceux qui n'ont pas de véhicule se limitent dans les marchés de production. Une portion des grossistes (18\%) préfère court-circuiter les collecteurs pour acheter directement aux producteurs leurs régimes à un prix plus bas que celui pratiqué par le collecteur. Ces derniers vont à la conquête des marchés les plus éloignés, que ce soit à l'intérieur du pays (Yaoundé, Douala) ou dans les pays voisins. Pour s'assurer les bas prix à l'achat, ils bravent les pistes rurales souvent mal entretenues. Ils paient au prix fort la location des camions pour acheter dans les villages les plus reculés à des producteurs appauvris et peu ou pas du tout informés sur les prix.

\section{Approvisionnement chez les détaillants}

$\mathrm{Au}$ niveau des détaillants, ils ne se contentent pas de la vente de plantain. Pendant les périodes de pénuries où le plantain devient trop cher dans les marchés de gros, certains détaillants préfèrent se tourner vers la vente d'autres vivres, comme le macabo et la patate douce. Ceux de Foumbot particulièrement ont mis en place une stratégie leur permettant d'acheter les régimes à moindre coût. Cette stratégie consiste à courtcircuiter les grossistes et les collecteurs pour acheter les régimes directement aux producteurs dans le marché de Baïgom.

\section{Stratégies pour faire face aux conditions de transport}

Le transport est un facteur déterminant de tout système de commercialisation. Le producteur, l'acteur de la première mise sur le marché, utilise plusieurs modes de transport pour pouvoir vendre son plantain. Les modes de transport des régimes sont assez variés (Figure 2). Dans les zones étudiées, le mode de transport le plus utilisé est le taxi (taxi brousse). Ici, il s'agit des Toyota Corolla, d'une capacité de 3 tonnes qui peuvent transporter jusqu'à 100 régimes par voyage. La préférence pour cette marque de voiture est imputable à sa capacité de charge, et le fait qu'elle permet de sillonner avec plus ou moins de facilité les différents points de collecte qui sont très souvent reliés par des pistes parfois mal entretenues. On peut lire sur la figure 2 que $76 \%$ des acteurs les utilisent pour transporter leur plantain. D'autres acteurs utilisent le «pousse-pousse » (porte-tout) et le transport à dos d'homme. Il s'agit respectivement des détaillants et des producteurs du fait de leur proximité au lieu de vente. Les camions et camionnettes sont utilisés par les grossistes qui font de longues distances pour aller revendre aux détaillants.

Les stratégies face aux barrières à l'entrée du marché

Stratégies des producteurs

Chez le producteur, les principales barrières limitant l'accès à la pratique de la culture de la banane-plantain dans la région de l'Ouest Cameroun sont le capital initial et l'accès à la terre. En ce qui concerne l'accès au capital initial, même si les producteurs de banane plantain dans la région de l'Ouest Cameroun pratiquent une agriculture de 
subsistance, il y existe néanmoins des activités qui nécessitent des moyens financiers dans certaines situations. En effet, l'acquisition de certains intrants, ou le recours à une main d'œuvre hors ménage peuvent nécessiter des moyens financiers assez importants. Et pourtant, ces dépenses peuvent s'avérer cruciales, notamment pour le lancement des activités agricoles. Quatre principales sources sont utilisées pour constituer le capital initial et financer les activités agricoles dans la région de l'Ouest Cameroun (Figure 3).

Dans l'ensemble des sites enquêtés, les sources du capital initial les plus importantes sont les revenus de la récolte précédente $(74 \%$ des producteurs) et l'aide familiale (16\% des producteurs). Il s'agit dans les deux cas, comme on peut bien s'en rendre compte, des capitaux propres que les paysans mettent en jeu pour se lancer dans les activités de production du plantain. Le constat frappant est que le crédit ne constitue pas un mode de financement des activités agricoles dans la région de l'Ouest Cameroun chez les producteurs de banane-plantain enquêtés.

Quant à l'accès à la terre, il semble constituer la barrière à l'entrée la plus importante dans la production de bananeplantain dans la région de l'Ouest Cameroun. La majorité des acteurs enquêtés $(57 \%$ des producteurs) confirment ce fait. La couche qui en souffre plus est celle des jeunes de moins de 30 ans. En effet, tant dans le droit moderne que dans le droit coutumier au Cameroun, l'accès à la terre n'est pas facile pour les plus jeunes, sauf à quelques exceptions près. Dans la production du plantain, la parcelle est en général cultivée par le propriétaire lui-même, les locations se font plus pour la culture des maraichères ou du maïs.

Stratégies des commerçants

Chez les commerçants (grossistes, collecteurs, détaillants), la barrière majeure reste le capital initial. Les sources de capital initial les plus importantes chez ceux-ci sont le crédit (40\%) soit auprès des établissements de microfinance, d'un usurier ou d'un proche et les tontines (37\%). Certains acteurs utilisent leur épargne personnelle pour se lancer dans la commercialisation du plantain (23\%).

Stratégies d'information sur le marché du plantain

Les acteurs enquêtés, surtout les producteurs agricoles affirment être informés de la situation du marché local du plantain. Ces informations sont obtenues auprès d'autres paysans et des commerçants. Certains producteurs préfèrent vendre leur production au niveau des marchés témoins spéciaux organisés par la Mission de régulation des approvisionnements des produits (MIRAP), et certains consommateurs informés s'approvisionnent directement dans ces marchés où le plantain est vendu au même prix que celui du bord champ. Cependant, le secteur est plus caractérisé par une asymétrie de l'information en défaveur du producteur. Le recours aux commandes par les collecteurs dans le processus d'approvisionnement est une pratique rencontrée rarement, 12,2\% seulement des enquêtés ont recours aux commandes auprès des producteurs malgré une utilisation croissante du téléphone portable dans les zones rurales. Les transactions se font essentiellement au comptant et en présence de deux acteurs, à savoir, l'acheteur et le vendeur.

\section{Rentabilité économique des activités de} commercialisation de la banane-plantain Charges supportées par les acteurs

Charges des producteurs

La charge unitaire par régime est de 635 FCFA, et la charge par $\mathrm{kg}$ de plantain est de 43 FCFA. Les frais de transport (75 FCFA) par régime représentent le montant moyen payé par le producteur pour transporter son plantain dans le marché de production et même parfois dans le marché de collecte, c'est le cas de Pont du Noun et Kouoptamo, le producteur paye 50 à 100 FCFA pour apporter son régime à Kouoptamo à parti du Pont du Noun ou de Bafounda-Mbouda. En période de pénurie, les routes sont peu praticables et le transport passe à $100 \mathrm{FCFA} /$ régime. En ce qui concerne le marché de Kouoptamo en particulier, une taxe de 25 FCFA est payée par les producteurs pour un régime qui entre 
dans le marché c'est la «taxe sur le plantain». Et dans le marché de Galim, une taxe de 100 FCFA est payée chaque jour de marché par le producteur qui vend ses régimes dans le marché, et le producteur vend en moyenne 4 régimes par semaine dans le marché de Galim, ce qui lui fait une taxe de $2 \mathrm{FCFA} / \mathrm{kg}$. La charge des producteurs est alors de 635 FCFA plus 25 FCFA, soit 660 FCFA par régime en période d'abondance et $45 \mathrm{FCFA} / \mathrm{Kg}$ dans les deux marchés de production et en période de pénurie ces charges sont de 660 FCFA +25 FCFA par régimes soit $47 \mathrm{FCFA} / \mathrm{kg}$.

Les Tableaux 1 et 2 respectivement présentent les récapitulatifs des charges de production des bananes plantains produites en période d'abondance et de pénurie. Zeugang (2013) trouvait plutôt que le producteur de banane plantain dans les zones périurbaines de Dschang supportait les charges de 658 FCFA/régime en vendant dans les marchés locaux.

\section{Charges supportées par les commerçants}

Les commerçants supportent aussi des charges dans leurs activités, le tableau 3 présente ces charges. Ce tableau révèle que les grossistes sont ceux qui supportent la plus grosse charge par régime $\left(\begin{array}{lll}15 & \text { à } & 22\end{array}\right)$ contrairement aux collecteurs et détaillants dont les charges sont moins importantes (5 à 7). On peut voir que le grossiste de l'axe A1 dépense 350 FCFA par régime pour le transport. Ceci équivaut à $95 \%$ des charges totales du grossiste par régime. Tchuisseu (2007) arrivait à la conclusion selon laquelle $84 \%$ des charges des «supers grossistes » impliqués dans le ravitaillement de la ville de Yaoundé en banane-plantain étaient destinées au transport. Ceci permet d'affirmer une fois de plus que le problème de transport demeure crucial dans la commercialisation des produits vivriers malgré les efforts que font les autorités pour améliorer les conditions d'approvisionnement des villes en vivres.

Charges supportées par les transporteurs

Le Tableau 4 montre que pour aller à Bamenda en partant de Mbouda, le transporteur supporte les charges de 44300 FCFA par semaine (un voyage aller simple). Ce qui correspond à 476 FCFA par régime, soit 33 FCFA $/ \mathrm{kg}$. Ceux qui se rendent à Bafoussam partant de Kouoptamo supportent, quant à eux, les charges de 37422 FCFA, soit $23 \mathrm{FCFA} / \mathrm{kg}$. L'automobile utilisée ici est une camionnette de marque Toyota Canter. Nous estimons son coût d'acquisition à 6500000 FCFA (deuxième main). Il s'amortit sur 6 années à hauteur de 1083333 FCFA par année et à raison 20833 FCFA par semaine sur l'axe A2, et sur 5 années sur l'axe $\mathrm{A} 1$ à raison de $25000 \mathrm{FCFA} /$ semaine et 1300000 FCFA/an, car le niveau de dégradation de la route est très avancé sur cet axe. Sur l'axe A2, on trouve 4 contrôles de police (sortie de Bafoussam par Sacta, à l'entrée de Foumbot, Baïgom et Kouoptamo), le transporteur dépense au moins 1000 FCFA/contrôle. Sur chaque axe se trouve un péage routier, le transporteur dépense 1000 FCFA en aller et retour pour le franchir.

Les marges nettes de commercialisation des acteurs

Le Tableau 5 montre que le producteur de l'axe A1 obtient une marge nette de 11 FCFA/kg en période d'abondance et celui de l'axe A2 reçoit une marge nette de 8 FCFA $/ \mathrm{kg}$ pendant la même période. Et pendant les périodes de pénuries, les marges des producteurs deviennent plus importantes. Elles sont de $31 \mathrm{FCFA} / \mathrm{kg}$ pour ceux de l'axe A1 et de $28 \mathrm{FCFA} / \mathrm{kg}$ pour ceux de l'axe A2 (Tableau 6). Cette augmentation de marge pendant la période de pénurie est due aux prix plus élevés.

Les grossistes ont la plus petite marge après le producteur évidement, ceci à cause de leur charge de transport qui est élevée. En effet, le coût de transport représente $95 \%$ des charges des grossistes, raison pour laquelle les grossistes multiplient les sources d'approvisionnement en régimes afin de faire le plein de la camionnette louée à cet effet. Les charges sont plus ressenties lorsque le véhicule loué est à moitié plein. Ceux de l'axe $\mathrm{A} 1$ achètent les régimes dans la région du Sud-Ouest Cameroun pendant que ceux de l'axe A2 se ravitaillent de plus en plus dans les zones périurbaines de Bafoussam. Leurs gains proviennent essentiellement du grand nombre de régimes qu'ils manipulent par 
semaine (93 régimes sur l'axe A1 et 110 régimes sur l'axe $\mathrm{A} 2$ ).

Le Tableau 7 révèle que les marges les plus importantes sont celles des détaillants. Respectivement $53 \mathrm{FCFA} / \mathrm{kg}$ sur l'axe $\mathrm{A} 1$ et 49 FCFA/ kg sur l'axe A2. Ceci s'explique par le fait que le détaillant pratique régulièrement le stockage des régimes de plantain pour les revendre mûrs, car le plantain mûr coûte beaucoup plus cher que le vert dans les marchés et surtout en période de pénurie.

Le prix de transport du plantain est payé en fonction du nombre de régimes à transporter. Les grossistes de l'axe A1 supportent une charge de 476 FCFA par régime, et $340 \mathrm{FCFA} /$ régime pour ceux qui vont sur l'axe A2. Cependant, le transporteur ne se déplace que s'il dispose d'un chargement pouvant faire le plein de son véhicule.

Le transporteur de Mbouda - Bamenda transporte le plantain à 700 FCFA pour 2 régimes, ce qui lui fait une marge de 224 FCFA par régime, soit $15 \mathrm{FCFA} / \mathrm{kg}$ et celui de Bafoussam à 500 FCFA les deux régimes, ce qui lui octroie une marge de 160 FCFA par régime, soit $12 \mathrm{FCFA} / \mathrm{kg}$.

\section{Facteurs limitant la performance du système de commercialisation \\ Difficultés rencontrées par les producteurs}

Le producteur de banane-plantain dans la région de l'Ouest Cameroun fait face à de nombreuses contraintes dans la conduite de ses activités de production. La figure 4 révèle l'importance des contraintes auxquelles il fait face, les plus importantes sont l'accès aux intrants et au crédit, pour respectivement $27 \%$ et $26 \%$ des producteurs.

\section{Difficultés rencontrées par les intermédiaires}

En ce qui concerne les intermédiaires (collecteurs, grossistes et détaillants), ils font aussi face à d'énormes difficultés dans la pratique du commerce de plantain. Les commerçants rencontrent trois difficultés majeures dans l'exercice de leurs fonctions. $\mathrm{Ce}$ sont respectivement le problème d'infrastructures de stockage, le vol dans les marchés et le transport (coût de transport et état des routes) cités respectivement par 34\%, $24 \%$, et $20 \%$ des intermédiaires (Figure 5).

Les détaillants font face au problème de stockage. En effet, les marchés sont dépourvus d'infrastructures de stockage adéquates pouvant garantir la qualité des produits stockés par les détaillants. Les tas de plantains sont stockés à l'air libre et à même le sol, protégés par une bâche. Les conséquences sont le pourrissement et la perte de la qualité hygiénique des régimes.

Les collecteurs et les grossistes, qui se déplacent régulièrement vers les lieux d'achat en zones rurales pour acheter les régimes, font face surtout aux problèmes de transport (état des routes, coût du transport, manutention inadaptée des régimes). Les routes sont peu praticables surtout en saison des pluies.

\section{Difficultés rencontrées par les transporteurs}

Les deux principales difficultés rencontrées par les transporteurs sont les tracasseries policières sur les routes et le mauvais état des routes rurales évoqués respectivement par près de $68 \%$ et $86,7 \%$ des transporteurs enquêtés. Cependant, les autres contraintes, moins évoquées mais non moins négligeables, sont le coût du carburant, le manque de plantain pendant les périodes de pénuries qui peut conduire à une baisse des activités et donc des revenus et la vétusté des engins (Tableau 8).

\section{Opportunités de développement des systèmes de commercialisation}

Malgré ces nombreuses contraintes, le commerce de la banane-plantain dans la région de l'Ouest Cameroun dispose d'atouts qui peuvent constituer un véritable levier pour son développement. Il s'agit notamment du dynamisme des acteurs impliqués dans la production, de la commercialisation du plantain dans la région et du niveau d'éducation moyen qui peut permettre de mieux cerner les stratégies novatrices en vue de l'amélioration des systèmes de commercialisation. Aussi, la forte demande sur le marché de la banane-plantain tant sur le plan national qu'au niveau de la sous-région serait un élément catalyseur. 


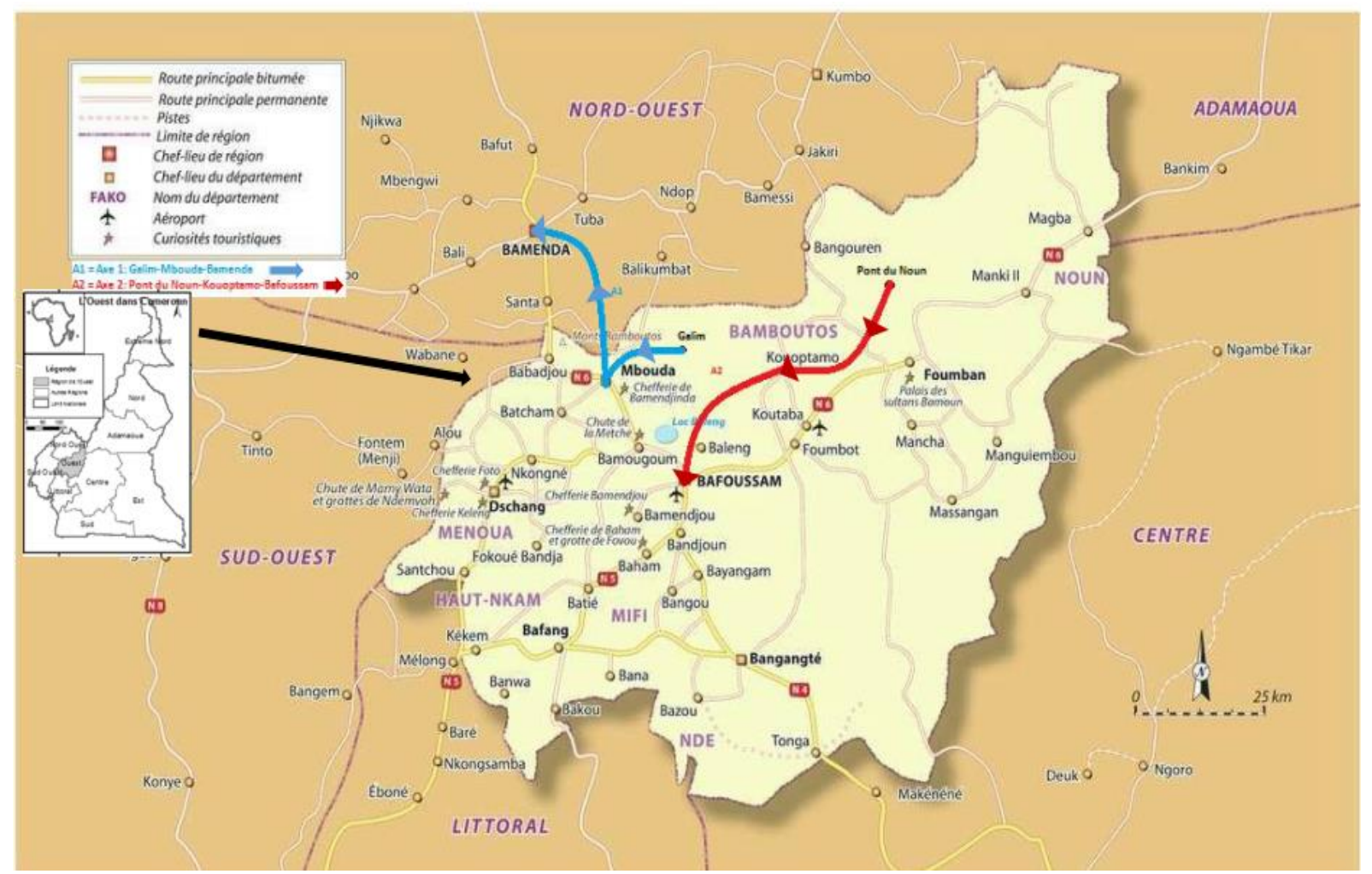

Carte 1: Les deux principaux axes de commercialisation de la banane-plantain à l'Ouest Cameroun.

Tableau 1 : Récapitulatif des charges de production en période d'abondance.

\begin{tabular}{lllllll}
\hline $\begin{array}{l}\text { Groupes } \\
\text { d'acteurs }\end{array}$ & \multicolumn{2}{l}{ Charges de productions } & \multicolumn{2}{l}{ Taxe sur le plantain } & \multicolumn{2}{l}{ Charges totales } \\
\cline { 2 - 6 } & FCFA/régime & FCFA/Kg & FCFA/régime & FCFA/Kg & FCFA/régime & FCFA/Kg \\
\hline $\begin{array}{l}\text { Producteur } \\
\text { de Galim }\end{array}$ & 635 & 45 & 25 & 2 & 660 & 45 \\
\hline $\begin{array}{l}\text { Producteur } \\
\text { du Pont du }\end{array}$ & 635 & 45 & 25 & 2 & 660 & 45 \\
\begin{tabular}{l} 
Noun \\
\hline
\end{tabular} & & & & & \\
\hline
\end{tabular}




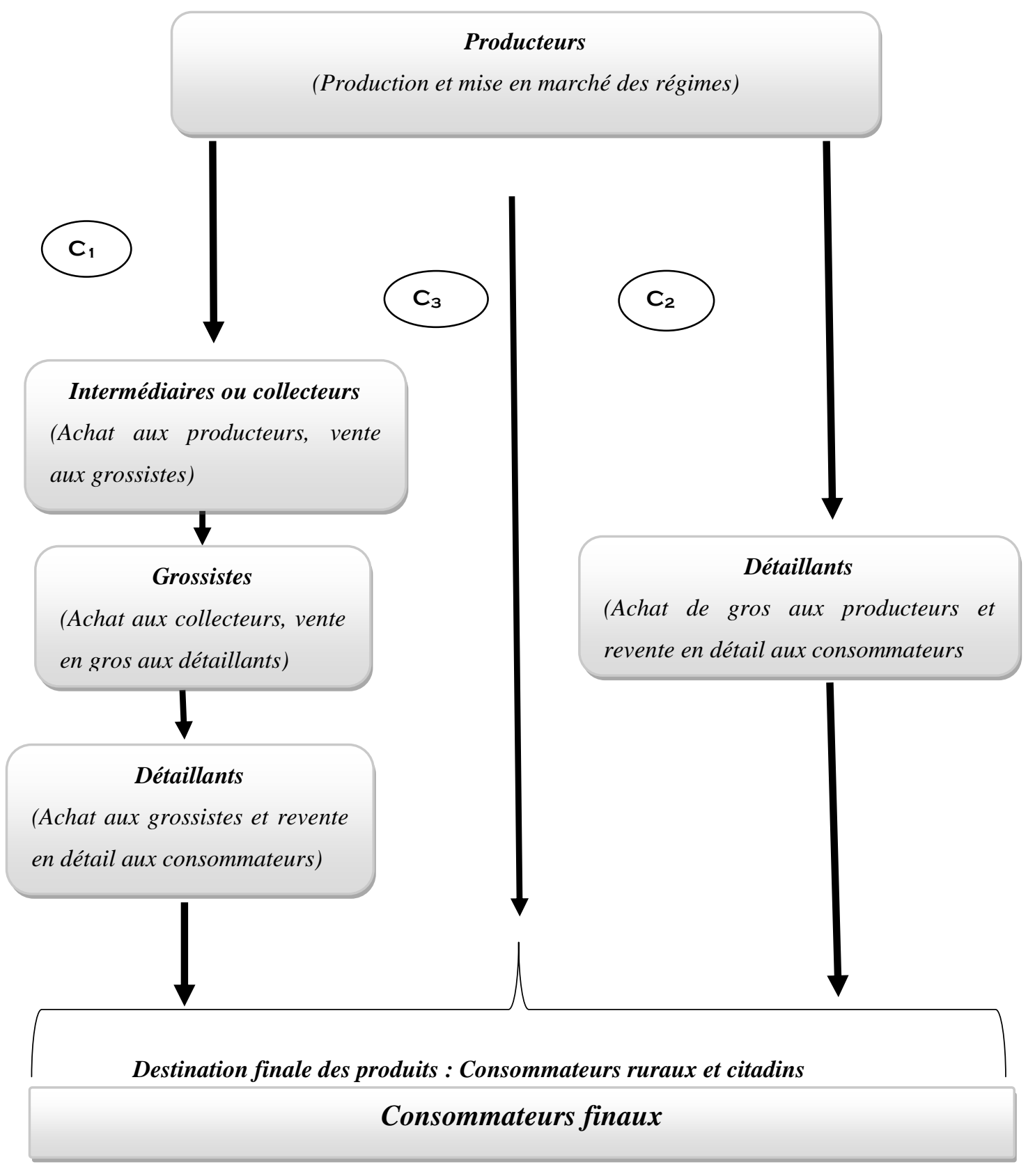

Légende : $\mathrm{C} 1, \mathrm{C} 2=$ Circuits indirects ; $\mathrm{C} 3=$ Circuit direct

$\mathrm{C} 1$ = Circuit long; $\mathrm{C} 2$ = Circuit court

Figure 1 : Circuits de commercialisation de la banane plantain. 


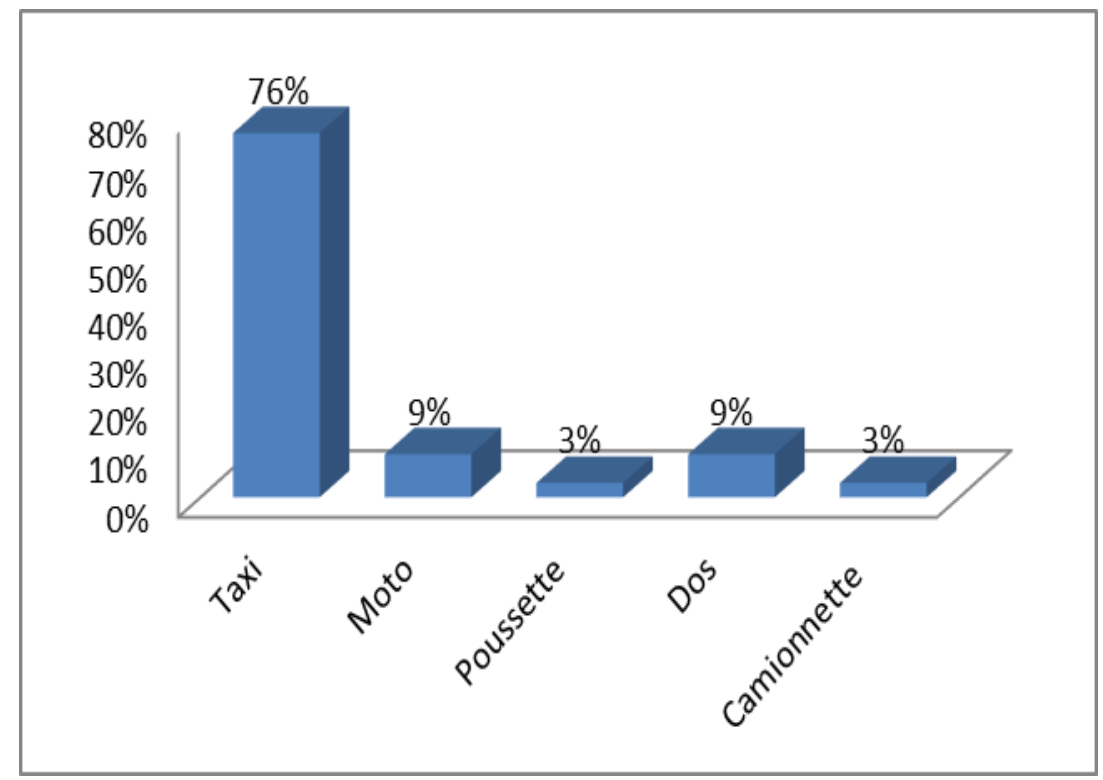

Figure 2 : Modes de transport des régimes.

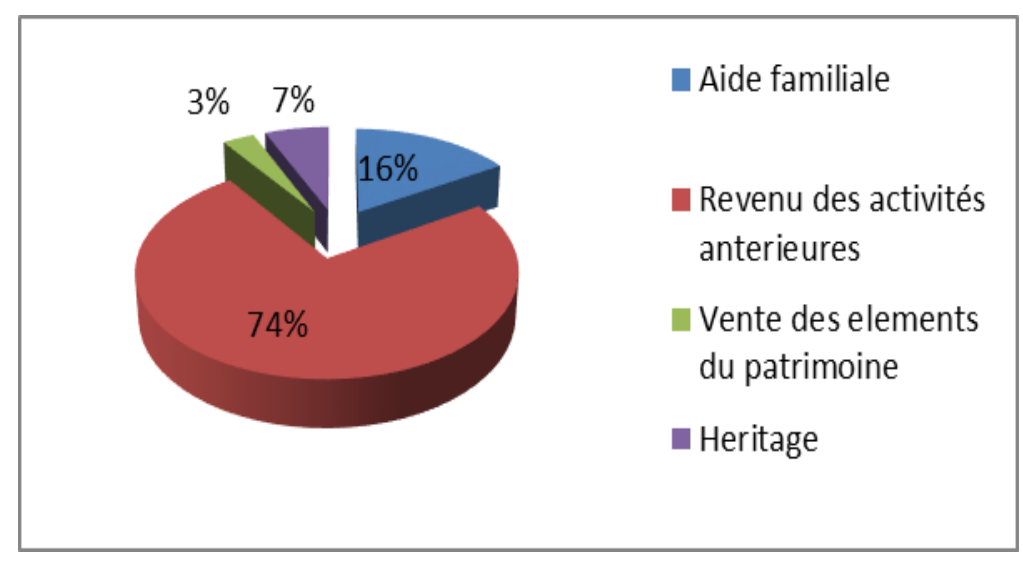

Figure 3 : Origine du capital initial des producteurs.

Tableau 2 : Récapitulatif des charges de production en période de pénurie.

\begin{tabular}{lllllll}
\hline $\begin{array}{l}\text { Groupes } \\
\text { d'acteurs }\end{array}$ & \multicolumn{2}{l}{ Charges de production } & \multicolumn{2}{l}{ Taxe sur le plantain } & \multicolumn{2}{l}{ Charges totales } \\
\cline { 2 - 7 } & FCFA/régime & FCFA/Kg & FCFA/régime & FCFA/Kg & FCFA/régime & FCFA/Kg \\
\hline $\begin{array}{l}\text { Producteur } \\
\text { axe A1 }\end{array}$ & 635 & 45 & 25 & 2 & 685 & 47 \\
\hline $\begin{array}{l}\text { Producteur } \\
\text { axe A2 }\end{array}$ & 635 & 45 & 25 & 2 & 685 & 47 \\
\hline
\end{tabular}


Tableau 3 : Charges supportées par les commerçants.

\begin{tabular}{lllllll}
\hline Groupes d'acteurs & \multicolumn{2}{l}{ Collecteurs } & \multicolumn{2}{l}{ Grossistes } & \multicolumn{2}{l}{ Détaillants } \\
\hline Marchés & Axe A1 & Axe A2 & Axe A1 & Axe A2 & Axe A1 & Axe A2 \\
\hline Transport & 100 & 50 & 300 & 250 & 50 & 50 \\
\hline Manutention & $/$ & $/$ & 10 & 10 & $/$ & $/$ \\
\hline Impôts et taxes & 4 & 25 & 5 & 4 & 16 & 14 \\
\hline Stockage & $/$ & $/$ & 2 & 2 & 9 & 8 \\
\hline Coût par régime & 104 & 75 & 317 & 216 & 75 & 72 \\
\hline Charge par kg & 7 & 5 & 22 & 15 & 5 & 5 \\
\hline
\end{tabular}

Tableau 4 : Les charges supportées par le transporteur par semaine en (FCFA).

\begin{tabular}{llcc}
\hline $\mathbf{N}^{\circ}$ & Rubriques & Charges en FCFA & \\
\hline & & Mbouda/Bamenda(A1) & Kouoptamo/Bafoussam (A2) \\
\hline 1 & Amortissement automobile & 25000 & 20832 \\
\hline 2 & Carburant & 15000 & 11090 \\
\hline 3 & Ticket & 300 & 500 \\
\hline 4 & Péage & 1000 & 1000 \\
\hline 5 & Tracasseries policières & 3000 & 4000 \\
\hline & Total & $\mathbf{4 4 ~ 3 0 0}$ & $\mathbf{3 7 4 2 2}$
\end{tabular}

Tableau 5 : Marge nette des producteurs selon les axes en période d'abondance (en FCFA/kg).

\begin{tabular}{lllll}
\hline $\begin{array}{l}\text { Axe de } \\
\text { commercialisation }\end{array}$ & Charge & Prix de vente & Valeur des pertes & Marge nette \\
\hline Axe A1 & 45 & 64 & 8 & 11 \\
\hline Axe A2 & 45 & 60 & 7 & 8 \\
\hline
\end{tabular}

Tableau 6 : Marge nette des producteurs selon les axes en période de pénurie (en FCFA/kg).

\begin{tabular}{lllll}
\hline $\begin{array}{l}\text { Axe de } \\
\text { commercialisation }\end{array}$ & Charge & $\begin{array}{l}\text { Prix de vente en } \\
\text { FCFA/kg }\end{array}$ & Valeur des pertes & Marge nette \\
\hline Axe A1 & 47 & 87 & 9 & 31 \\
\hline Axe A2 & 47 & 83 & 8 & 28 \\
\hline
\end{tabular}


Tableau 7 : Marges nettes des commerçants en FCFA/kg selon les axes en période de pénurie.

\begin{tabular}{|c|c|c|c|c|c|}
\hline $\begin{array}{l}\text { Axe de } \\
\text { commercialisation }\end{array}$ & $\begin{array}{l}\text { Prix } \\
\text { d'achat }\end{array}$ & Prix de vente & Charges & $\begin{array}{l}\text { Valeur des } \\
\text { pertes }\end{array}$ & $\begin{array}{l}\text { Marge } \\
\text { nette }\end{array}$ \\
\hline Collecteur A1 & 87 & 140 & 7 & 4 & 42 \\
\hline Collecteur A2 & 83 & 136 & 5 & 5 & 43 \\
\hline Grossiste $\quad$ A1 & 140 & 199 & 21 & 6 & 32 \\
\hline Grossiste A2 & 136 & 190 & 14 & 6 & 34 \\
\hline Détaillant A1 & 199 & 265 & 5 & 8 & 53 \\
\hline Détaillant A2 & 190 & 249 & 5 & 5 & 49 \\
\hline
\end{tabular}

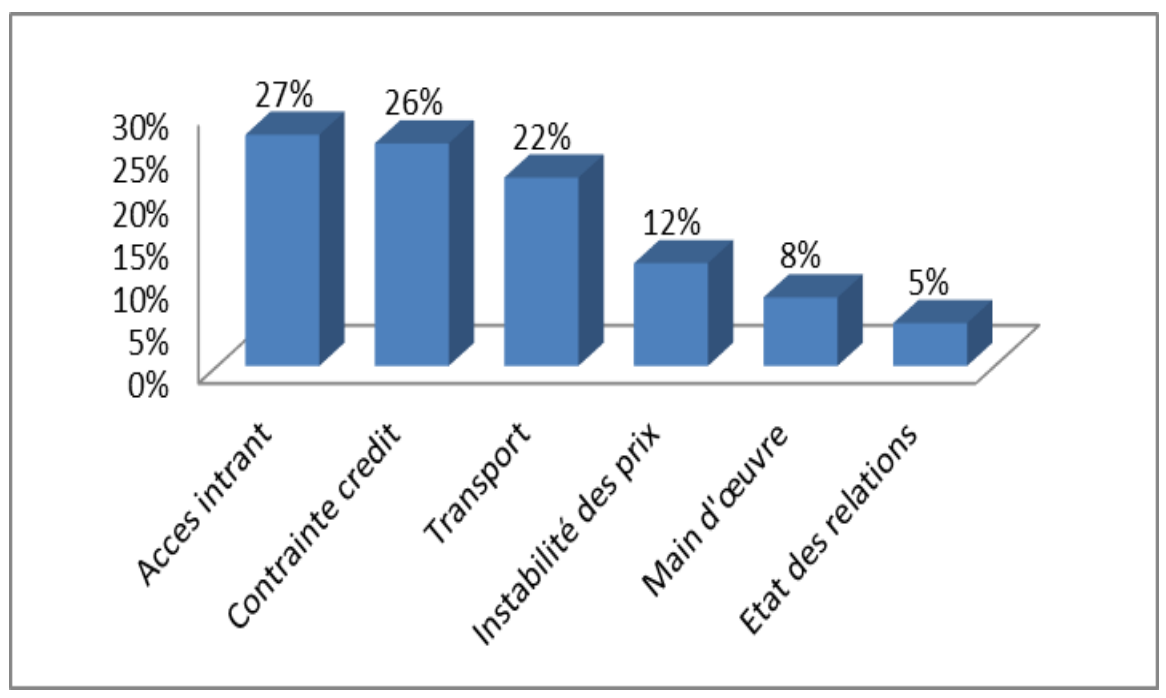

Figure 4 : Contraintes des producteurs.

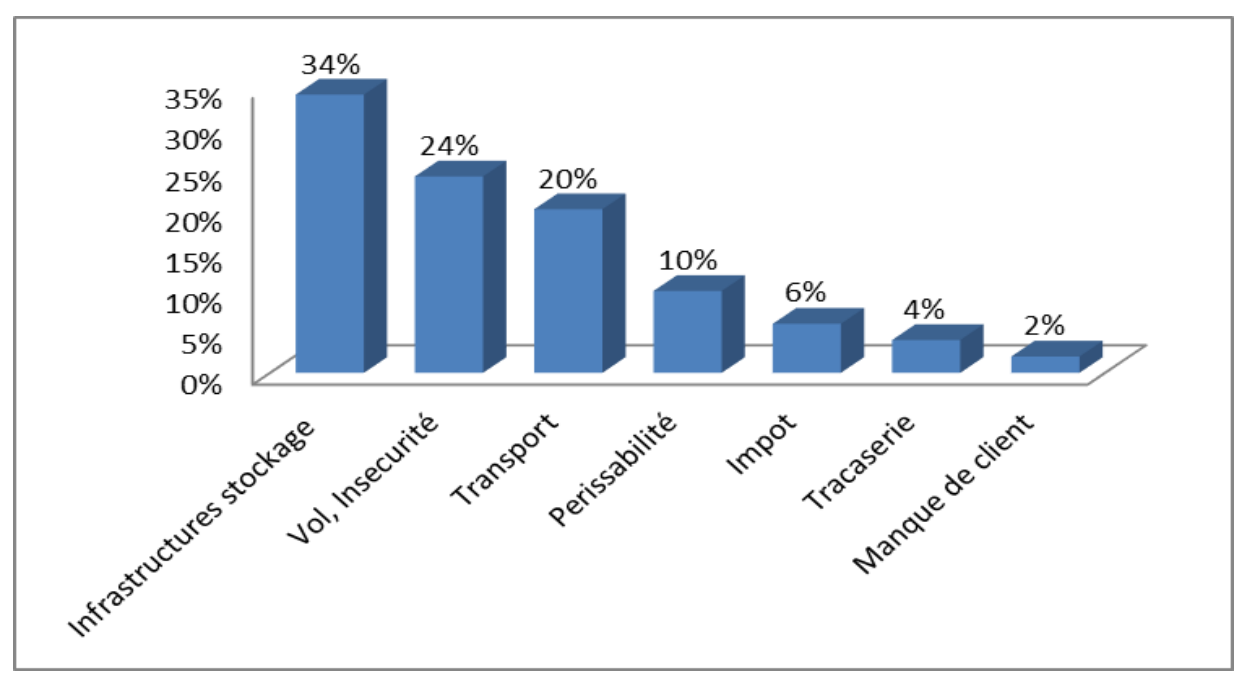

Figure 5 : Difficultés rencontrées par les commerçants. 
Tableau 8 : Difficultés rencontrées par les transporteurs.

\begin{tabular}{lc}
\hline Difficultés rencontrées & Pourcentage (\%) \\
\hline Mauvais état des routes & 86,7 \\
\hline Tracasseries policières & 68 \\
\hline Cherté du carburant & 10 \\
\hline Vétusté des véhicules & 7 \\
\hline
\end{tabular}

\section{DISCUSSION}

Il existe une diversité d'acteurs impliqués dans le système de commercialisation de la banane plantain dans la région de l'Ouest Cameroun. A la base de la chaine, on a le producteur qui constitue l'acteur principal qui conditionne même l'existence des autres catégories d'acteurs. En effet, c'est lui qui produit et met dans le circuit de commercialisation la bananeplantain. L'étude a montré que cette catégorie reste dominée par les hommes d'un âge suffisamment avancé, ce qui montre sans doute qu'on a à faire à une activité qui nécessite beaucoup d'efforts physiques, et surtout une expérience significative. L'étude met en exergue une seconde catégorie d'acteurs qui est constituée des intermédiaires (les collecteurs, les grossistes, les détaillants et dans une moindre mesure, les transporteurs). $\mathrm{Ce}$ sont donc ces intermédiaires qui assurent l'acheminement des produits depuis les zones de productions jusqu'aux marchés de consommation. Exception faite du transport qui reste l'activité exclusive des hommes, les autres intermédiaires que sont les collecteurs, détaillants et grossistes sont majoritairement des femmes qui se révèlent être des fines négociatrices. Ces résultats confirment ceux des études antérieures qui présentent les femmes comme très actives dans la commercialisation des produits agricoles au Cameroun (Folefack et al., 2017). Le fait que les acteurs impliqués dans cette filière soient pour la plupart mariés, avec un niveau scolaire relativement bas (niveau primaire pour la plupart), montre que l'activité requiert un certain niveau de responsabilité, et d'expérience, mais que le niveau scolaire n'est pas une composante déterminante de l'efficacité des rôles joués par ces derniers.

L'analyse du circuit de commercialisation de la banane plantain dans la région de l'Ouest Cameroun a permis d'identifier les deux axes les plus importants qui sont l'axe Galim $\rightarrow$ Mbouda $\rightarrow$ Bamenda, et l'axe Pont du Noun $\rightarrow$ Kouoptamo $\rightarrow$ Bafoussam. La Figure 1 montre que le producteur qui est à la base peut décider, en fonction de ses moyens, de vendre soit bord champ, soit au marché local d'approvisionnement ou directement aux détaillants dans les centres urbains. Quant aux collecteurs, ils achètent le plantain bord champ, réalisent plusieurs services, notamment la collecte auprès des petits producteurs et le regroupement dans une zone plus accessible aux grossistes qui se limitent généralement aux points de ravitaillement définis préalablement avec les collecteurs. La différence entre les grossistes et les collecteurs c'est que ce sont généralement les collecteurs qui sillonnent les différentes exploitations car ils maitrisent bien les lieux et entretiennent d'étroites relations avec les producteurs, ce qui n'est pas toujours le cas pour les grossistes. Ces collecteurs jouent différents rôles essentiels pour les producteurs isolés. Ils s'organisent pour venir chercher les produits en des endroits éloignés et peu accessibles, compte tenu de l'état des routes. Ils doivent pour rester informés, organiser des tournées, louer s'ils n'en possèdent pas un véhicule pour transporter les produits. Les transporteurs se déplacent lorsqu'ils sont sollicités soit par les collecteurs soit par les grossistes, ce qui se fait très souvent la veille ou le jour du marché. Dans l'exécution de leurs tâches, ils sont aidés par les 
manutentionnaires qui ont pour rôle de charger les régimes de plantain dans le véhicule. Ceux-ci sont parfois les «motos boys» (manutentionnaire accompagnant le chauffeur) ou des personnes payées sur place uniquement pour la circonstance. Pour évaluer la performance des circuits de commercialisation au Niger, Malam Boukar et al., (2016) identifie trois démarches méthodologiques complémentaires: $\quad \mathrm{La}$ première permet $d$ 'identifier les produits agricoles les plus rentables selon l'avis des producteurs, la seconde permet de déterminer les principaux produits agricoles de cuvettes à travers le "test de Kendall " et la troisième permet de calculer la rentabilité économique des produits à travers des indicateurs de performance.

Les stratégies d'acteurs impliqués dans la production et la commercialisation de la banane-plantain varient en fonction des types d'acteurs. La stratégie d'approvisionnement chez les producteurs repose essentiellement sur l'approvisionnement en semences. Dans bien de cas, le producteur se positionne également comme le principal, sinon l'unique pourvoyeur de semences. Ceci démontre la fragile collaboration qui existe entre eux et les structures de production des semences. Beaucoup déclarent ne pas connaître l'existence du CARBAP. Rares sont ceux qui connaissent le Programme de Relance de la Filière Plantain (PRFP) et peinent à adopter leurs semences. Pour les collecteurs et grossistes, la stratégie se résume à user de tous les astuces possibles pour acquérir le produit au moindre coût, pour ainsi maximiser les profits. Certains collecteurs et grossistes achètent les régimes en champs avant qu'ils ne soient récoltés, cela arrive lorsque le producteur a un besoin urgent d'argent. Pour les producteurs, les stratégies développées face aux barrières à l'entrée du marché se résument en l'acquisition du capital financier et foncier. Les paysans sont parfois contraints d'exploiter des petites superficies par manque des moyens financiers. Les institutions de microfinance octroient de plus en plus des crédits aux commerçants citadins. L'agriculture ne semble pas encore intéresser ces institutions malgré le besoin exprimé, surtout que les paysans ne disposent pas généralement des garanties fiables à présenter pour bénéficier d'un crédit. L'accès à la terre est particulièrement difficile pour les plus jeunes, sauf à quelques exceptions près soit de ceux résidant en milieu rural et dont le capital foncier des parents est assez conséquent ou ceux ayant hérité d'une vaste superficie de terres. La parcelle est en général cultivée par le propriétaire lui-même. Les stratégies d'information sur les marchés sont de plus en plus assurées par le téléphone portable qui devient progressivement un outil indispensable dans le système d'information en milieu rural, en lieu et place du bouche à oreille d'autrefois.

Les facteurs limitant la performance des systèmes de commercialisation s'observent à plusieurs niveaux. Chez les producteurs, ceux-ci ne disposent pas d'assez de moyens financiers lorsqu'il faut engager les dépenses importantes dans la production agricole. L'unique intrant se limite généralement à la semence dans la plupart des cas. Les conditions de transport et l'instabilité des prix ont également été évoquées parmi les contraintes les plus ressenties. Pour les commerçants grossistes, le problème d'infrastructures de stockage, le vol dans les marchés, le coût de transport et l'état des routes sont dominants. Les contraintes de transport des produits vivriers en général et du plantain en particulier dans la région de l'Ouest Cameroun peuvent être à la base des pertes qui se produisent pendant ce transfert. Les conditions de transport sont donc caractérisées par les tracasseries, le mauvais état des routes, les pannes fréquentes qui allongent la durée du voyage, les surcharges des camions, l'absence de conditionnement et une manutention inadaptée. A partir de ces résultats, nous constatons que les contraintes de développement de la filière dans cette région sont restées les mêmes, car Nkendah et Temple en 2003 évoquaient déjà les mêmes contraintes. Cela montre que des actions de développement mis en place n'ont pas amélioré la situation. Il est donc clair qu'il faudrait repenser les stratégies de production 
et de commercialisation de la filière, en renforçant les synergies entre les différents acteurs impliqués et le mis en place d'un système d'information efficace orienté vers le développement de la filière.

\section{Conclusion}

L'étude de la commercialisation d'un produit vivrier dans une région où la majorité de la population vit dans le milieu rural et pratique l'agriculture constitue une activité essentielle pour le bien-être général. Le présent article a permis de ressortir les profils sociodémographiques des acteurs impliqués dans la production et la commercialisation de la banane-plantain dans la région de l'OuestCameroun. Un certain nombre d'informations concernant les axes et circuits de commercialisation ont été mis en exergue de même que les stratégies des acteurs, les charges et les marges bénéficiaires des acteurs. Il ressort que les activités de production sont majoritairement assurées par les hommes, tandis que les femmes sont plus impliquées dans la vente (collecte, vente en gros et détail). Le transport, quant à lui, reste une exclusivité masculine. Deux axes de commercialisation ont été identifiés: l'axe A1, Pont du Noun-Kouoptamo-Bafoussam et l'axe A2, Galim-Mbouda-Bamenda. Les acteurs de l'axe A1 supportent les plus grosses charges et les marges les plus importantes sont celles des détaillants. La production et la commercialisation de la banane-plantain s'avère rentable pour les acteurs impliqués, mais le secteur est caractérisé par une asymétrie de l'information en défaveur du producteur. Il est recommandé aux acteurs de négocier des contrats de commercialisation afin de réduire les aléas liés à la vente du plantain.

\section{CONFLITS D'INTERETS}

Les auteurs déclarent que dans la cadre de ce travail, il n'existe aucun conflit d'intérêts.

\section{CONTRIBUTIONS DES AUTEURS}

GHFF, AB et DPF sont les principaux investigateurs de cet article. Ils ont contribué à la conception méthodologique, l'analyse des données et la rédaction du manuscrit; IT a contribué à la collecte des données sur le terrain et l'analyse statistique; PN a contribué à la relecture du manuscrit.

\section{REMERCIEMENTS}

Les auteurs tiennent à remercier particulièrement le staff du projet C2D/PAR Plantain pour l'attention particulière accordée à la réalisation et au financement de ce travail.

\section{REFERENCES}

Bouafou KGM, Konan BA, Kouame KG, Kati-Coulibally S. 2012. Les produits et sous-produits du bananier dans l'alimentation animale. Int. J. Biol. Chem. Sci., 6(4): 1810-1818. DOI: http://dx.doi.org/10.4314/ijbcs.v6i4.36.

Folefack DP, Fongang FGH, Mohamadou AK, Ebongue JP, Bikoi A, Noupadja P. 2017. Analysis of the plantain supply system of markets in the city of Douala. Journal of Economics and Sustainable Development. 8(6).

Fongang FGH, Folefack DP, Pane PZ, Bikoi A, Noupadja P. 2016. Transformation et commercialisation des chips de banane plantain au Cameroun : une activité artisanale à forte valeur ajoutée. Int. J. Biol. Chem. Sci., 10(3): 1184-1198. DOI http://dx.doi.org/10.4314/ijbcs.v10i3.23 .

IRAD/CARBAP. 2012. Amélioration durable de la productivité et de la compétitivité de lade la filière plantain au Cameroun par l'utilisation des technologies innovantes. 53p.

Lepengue AN, Mouaragadja I, Dick E, Mbatchi B, Ake S. 2010. Amélioration de la durée de conservation des bananes plantain aux températures ambiantes. Int. J. Biol. Chem. Sci., 4(3): 730-737. http://ajol.info/index.php/ijbcs

Lothoré A, Delmas P. 2009. Accès aux marchés et commercialisation des produits agricoles, valorisation des initiatives des producteurs. Document collectif, inter réseau Développement rural, pp 23-24.

Malam Boukar AK, Tidjani AD, Yamba B, Lebailly P. 2016. Performance et circuit de commercialisation des principaux 
produits agricoles des cuvettes oasiennes du département de Gouré (Niger). Int. J. Biol. Chem. Sci., 10(5): 2202-2214. DOI: http://dx.doi.org/10.4314/ijbcs.v10i5.21.

MINADER. 2012. Programme de relance filière plantain. Séminaire de renforcement des Capacités des producteurs Ouest, 15-17 juin.

Nkendah R, Akyeampong E. 2003. Données socioéconomiques sur la filière plantain en Afrique centrale et de l'ouest. Infomusa, 12(1): 8-13.

Nkendah R, Beatric C, Nzouessim BC, Temple L. 2007. Economic analysis of the spatial integration of plantain markets in Cameroon. African journal of Economic Policy, 14(1): 57-83.

Nkendah R, Temple L. 2003. Pression démographique et efficacité technique des producteursdes producteurs de banane plantain de l'ouest Cameroun. Cahiers D'agricultures (Fra), 125(5): 333-339.

Nkendah R, Temple L. 2003. Pression démographique et efficacité technique des producteurs de banane plantain de l'Ouest-Cameroun. Cahiers Agricultures, 12(5) : 333-339.

Odah K, Aziadekey M, Tozo K, Akpavi S, Koukouma R, Guelly A, Kokou K,
Assignon K, Akogo Y, Aidam A, Akpagana K, Kenny L, Ait-Oubahou A, Zinsou C, Gbeassor M. 2013. La diversité génétique des bananiers plantains cultivés dans la zone Ouest de la Région des Plateaux au Togo. Int. J. Biol. Chem. Sci., 7(5): 1910-1918. DOI: http://dx.doi.org/10.4314/ijbcs.v7i5.11.

Tchuisseu TRA. 2007. Analyse de la filière banane plantain d'approvisionnement de la Ville de Yaoundé. Mémoire de fin d'études. FASA. Université de Dschang. Cameroun. 82p.

Unctad. Bananes. 2011. http://www.unactad.org/infocomm/franc ais/banane/marche.htm Consulté le 13 septembre 2015.

Wandji AA. 2015. Dynamique des marchés transfrontaliers de la banane plantain de la vallée du Ntem (Sud Cameroun). Mémoire de Master II, ESSEC, Université de Douala.

Zeugang S. 2013. Impact de la production et la commercialisation de la banane plantain sur le revenu du producteur dans la zone périurbaine de Dschang, région de l'Ouest-Cameroun. Mémoire présenté en vue de l'obtention du diplôme d'ingénieur agronome. FASA. Université de Dschang. 101p. 